\title{
Modelling Water: A Lifetime Enigma
}

\author{
John F. Ouyang and Ryan P. A. Bettens*
}

\begin{abstract}
The first attempt to describe water dates back to 1933 with the Bernal-Fowler model and it would take another forty years before the first computer simulation of liquid water by Barker and Watts in 1969. Since then, over a hundred different water models have been proposed. Despite being widely studied, water remains poorly understood. Examining the evolution of water models, we identified three distinct philosophies in water modelling, namely the employment of effective point charges in pioneering empirical models, the incorporation of polarization to describe many-body inductive effects and the extensive use of $a b$ initio calculations to describe short-range effects. In doing so, we can appraise the current understanding of water and identify attributes that a water model should possess to capture the intricate interactions between water molecules.
\end{abstract}

Keywords: Force field $\cdot$ Molecular dynamics $\cdot$ Polarizable $\cdot$ Potential energy surface $\cdot$ Water models

\section{Introduction}

Considering the rich history of water modelling, it would be prudent to ask why scientists across different disciplines are enthralled by water. An obvious motivation would be its abundance which suggests that water is undeniably important in the grand scheme of nature. The strange properties associated with water also spur academic curiosity to unravel the mysteries behind this small molecule. Most importantly, deciphering the interactions between water molecules would lead to basic understanding of intermolecular forces, which govern many dynamic processes in nature.

Given its ubiquity in nature, water has been the subject of extensive research. On Earth, water is the central solvent for naturally occurring chemical processes. In particular, water is the medium for biochemical interactions, widely recognized as the 'matrix of life'. ${ }^{[1]}$ Its place in biology goes beyond a passive solvent, having many active roles in molecular biology. [2-4] Water-mediated hydrogen bonding provides exchangeable and extensible linkages to manoeuvre the peptide backbone during protein folding, allowing proteins to achieve their active conformation rapidly. ${ }^{[5]}$ Hydration changes can induce modification in DNA conformation and interfacial water possesses a unique sequence-dependent hydration structure, acting as a 'hydra-

\footnotetext{
*Correspondence: Dr. R. P. A. Bettens Department of Chemistry National University of Singapore

3 Science Drive 3, Singapore 117543

E-mail: chmbrpa@nus.edu.sg
}

tion fingerprint' for the recognition of the DNA sequence. ${ }^{[6]}$ On a cosmic scale, detection of water vapour in the atmosphere of an extrasolar gas-giant planet suggests that the presence of water is common in gasgiants. ${ }^{[7]}$ Closer to home, studies on the isotopic composition of water in meteorites help us gain insights about the origins of the early solar system. ${ }^{[8,9]}$ Interestingly, most water in the universe exists as different forms of amorphous ice and their transitions in cold dense interstellar molecular clouds causes radical recombination, resulting in the synthesis of complex organic molecules. ${ }^{[10]}$ The role of water in many chemical and biological processes that are responsible for sustaining life, is the driving force behind understanding its behaviour under different conditions, and in various environments.

Being one of the most studied substances, many physical properties of water are accepted as international standards such as its triple point and density. ${ }^{[11]}$ Even so, many of these physical properties are considered anomalous as they contradict the general theories of the liquid state of matter. The most widely known property would be the maximum density of water at $4{ }^{\circ} \mathrm{C}$, making water the only liquid to expand upon cooling. Other anomalies include the non-monotonic behaviour of its isothermal compressibility and specific heat. ${ }^{[12,13]}$ Furthermore, water exhibits a very high boiling point and dielectric constant for a simple liquid. Although the aforementioned anomalies were known for some time, new anomalous behaviours are constantly uncovered. It was found that supercooled water becomes more diffusive as pressure is increased to about $200 \mathrm{MPa}$ at room temperature. ${ }^{[14]}$ Also, the discovery of another supercooled liquid water state at $150 \mathrm{~K}$ challenges the notion of a single supercooled regime at ambient pressure ${ }^{[15]}$ and this newly discovered supercooled state may lead to the identification of a possible second critical point in supercooled confined water. ${ }^{[16]}$ If the liquid state is strange, the solid state would be bizarre with water having fifteen known forms of ice, many of which were only recently discovered. ${ }^{[17,18]}$ It is ironic that while better technology has allowed us to probe the properties of water further, these observed phenomena can exacerbate confusion as they remain unexplained.

The wealth of knowledge on water, many of which deemed anomalous, imposes severe tests on any newly proposed water model. Despite being a chemically simple molecule, water is notoriously hard to model. First, water can give rise to extensive hydrogen bonding networks. [19] As early as 1920, hydrogen bond is first suggested to occur in water ${ }^{[20]}$ and it is commonly agreed that these fleeting hydrogen bonds makes water unique from most other liquids. Dimer interactions are dominated by a deep minimum at the hydrogen-bonded configuration, ${ }^{[21-23]}$ implying that certain configurations are preferred in water clusters and bulk water. The strong directionality of hydrogen bonding is the reason for the inclusion of explicit water molecules in simulating water-mediated processes such as protein folding. ${ }^{33]}$ However, the hydrogen bond minimum is not overly stabilising, making dynamic hydrogen bonding rearrangements possible in bulk water. ${ }^{[19]}$ Second, the description of water is complicated by strong non-additive inductive effects that manifest in water due to the large dipole and polarizability of water. Such inductive effects can enhance the dipole moment of water molecules by more than $60 \%$ in the condensed phase. [24] This is further complicated by the fact that the introduction of polarizability can be rather deceptive, ${ }^{[25]}$ compounded by 
reasons which will be covered in Section 3.1. All in all, water is especially sensitive to how the forces between molecules are described and thus demand a thorough and basic understanding of intermolecular forces.

\section{Water Models}

The Bernal-Fowler (BF) model can be considered the first realistic water model, describing water as a collection of point charges and a repulsion-dispersion term. ${ }^{[26]}$ A similar representation would be used later in the first Monte Carlo simulation of water by Barker and Watts ${ }^{[27]}$ and the first Molecular Dynamics (MD) simulation of water by Rahman and Stillinger.[28] Since the first computer simulation of water, a myriad of water models, exceeding a hundred to date, have been proposed. While there already exist several excellent reviews on the progress of modelling water, ${ }^{[29-33]}$ we still wish to survey the water modelling scene with the aim of highlighting the qualities of a good water model.

In the aforementioned reviews, water models are categorized based on (i) the interaction between water monomers and (ii) the treatment of water monomers. Polarizable models treat many-body inductive effects explicitly using point polarizabilities whereas non-polarizable models describe this polarization in an averaged manner in the pairwise interactions. Rigid water models constrain the intramolecular degrees of freedom, typically to that of the vibrational averaged geometry while flexible counterparts relax all degrees of freedom. Due to $a b$ initio calculations ap- proaching experimental accuracy, water models can also be classified based on the nature of the data ( $a b$ initio or experimental or both) used to parameterise the model.

Instead of following these traditional and possibly restricting classifications, we analysed the evolution of water models and broadly identified three distinct philosophies in the saga of water modelling, namely the employment of enhanced point charges in pioneering models to effectively describe induction in a pairwise potential, the incorporation of polarization in later models to describe explicitly the many-body inductive effects and the extensive use of $a b$ initio data in state-of-the-art models to accurately describe water-water interaction at all ranges (Fig. 1). Water models are not necessarily grouped based on chronological order as these demarcations represent distinct principles of water modelling rather than actual time periods. In doing so, we have alluded to the long history of water modelling and its coming of age.

\subsection{Pioneering Empirical Water Models}

This class of water models has its origins in legacy water models, aimed at describing water with a low computational cost and thus often utilise a rigid water monomer. Similar to the BF model, these models are empirical and non-polarizable, using point charges to represent electrostatics and a Lennard-Jones term for dispersion and repulsion. Induction effects are effectively described by increasing the point charges to simulate an enhanced dipole moment found in the condensed phase. Parameters are fitted to reproduce macroscopic experimental data such as the liquid density and heat of vaporization. The reliance on experimental data can be reconciled by noting that these models flourished in the 1980s while highly accurate $a b$ initio tools such as the Coupled-Cluster Single and Double, and perturbative treatment of Triple excitations [CCSD(T)] level of theory ${ }^{[34]}$ and Dunning correlation basis sets ${ }^{[35]}$ were only developed in 1989 and became computationally feasible many years later. Consequently, these models only work well at reproducing macroscopic properties of the condensed phase near the conditions under which they are parameterized, typically ambient pressure and temperature, and are targeted towards applications such as biomolecular simulations rather than basic scientific enquiry about the anomalous properties of water. The low computational cost associated with these models would make them remain the preferred choice for the most computationally demanding applications. For example, the TIP3P model is the default water model used in the CHARMM force field for biomolecular simulations. ${ }^{[36]}$ One of the earliest water models in this class is the MCY model, [37] well-known for being constructed entirely from ab initio Hartree-Fock (HF) calculations. We will also look further into two families of these pioneering water models, namely the TIP $n \mathrm{P}$ and SPC water models.

\subsubsection{TIPnP Family}

First developed by Jorgensen in 1981 as the Transferable Intermolecular Potential functionS (TIPS), ${ }^{[38]}$ it was later refined into the TIP3P and TIP4P model[39] which most water scientists are familiar

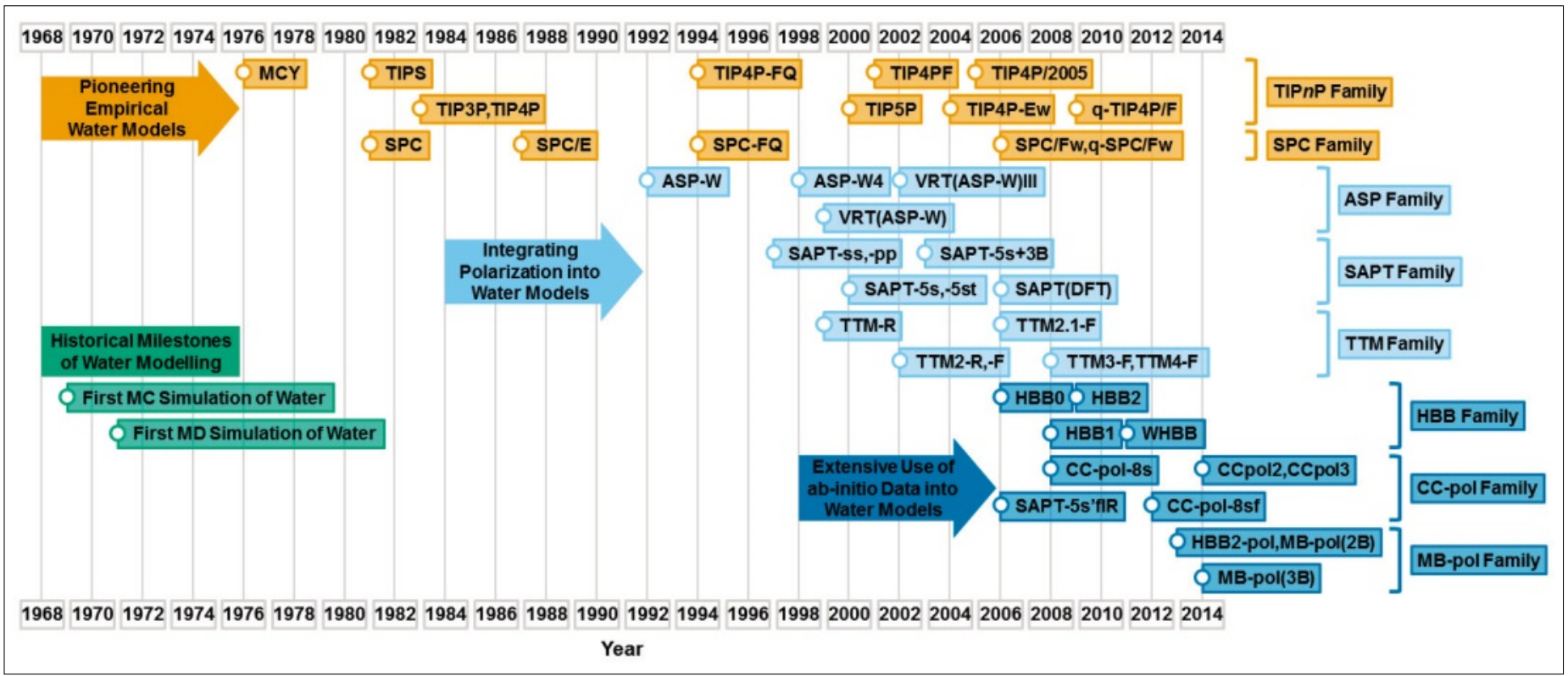

Fig. 1. Timeline showing the year of implementation of various water models reviewed in this paper. Water models are grouped (using different colour schemes) according to the three distinct philosophies of modelling water identified. Within each class of water models, the models are further subdivided into different families of water model that share similar traits. 
with. Here, the $n \mathrm{P}$ refers to the number of point sites in the model where point charges and/or Lennard-Jones terms are placed. In the simplest case, the atomic sites were used as seen in TIP3P. An additional M-site along the $\mathrm{HOH}$ angle bisector is introduced in TIP4P to displace the negative charge towards the hydrogens as placing the negative charge on the oxygen would lead to an excessively high dipole moment. ${ }^{[27]}$ In an attempt to describe inductive effects, TIP4P-FQ (FQ for fluctuating charge) was introduced where the point charges fluctuate in response to the environment to equalize the electronegativities of the sites. ${ }^{[40]}$ Later, Mahoney and Jorgensen would introduce more TIP $n \mathrm{P}$ variants, namely TIP5P ${ }^{[41]}$ and TIP4PF. ${ }^{[42]}$ TIP5P replaced the M-site with two tetrahedral negative charges to mimic the lone pairs on water but this resulted in a overly structured water in simulations. TIP4PF is a flexible version of TIP4P where intramolecular stretching and bending are described by quadratic terms and the same study showed that the inclusion of quantum effects improve the predictions made by this flexible water model.

Surprisingly, the models mentioned thus far truncate long-range electrostatics at a certain cut-off distance. The TIP4PEw model is designed for use with Ewald techniques to account for long-range electrostatics, commonly employed in biomolecular simulations. ${ }^{[43]}$ Numerous other parameterization attempts were made, such as TIP4P/2005 ${ }^{[44]}$ and q-TIP4P/F, ${ }^{[45]}$ which are optimised to better reproduce the thermodynamic properties of water and to account for quantum effects respectively.

\subsubsection{SPC Family}

Apart from the TIPnP family, another family of water models is the Single Point Charge (SPC) model, which only uses the three atomic sites to place point charges and/or Lennard-Jones terms. ${ }^{[46]}$ Simple values were used for its parameters such as $1.0 \AA$ for the $\mathrm{O}-\mathrm{H}$ bond length and an ideal tetrahedral angle of $109.5^{\circ}$ instead of the experimental gas-phase values used in TIP4P. Shortly, the improved SPC/E model was proposed to account for polarization self-energy. ${ }^{[47]}$ Similar to TIP4P-FQ, SPCFQ was introduced to incorporate induction effects. ${ }^{[40]}$ Likewise, flexible monomer versions such as $\mathrm{SPC} / \mathrm{FW}_{\mathrm{W}}{ }^{[48]}$ and variants parameterized to account for quantum effects, such as the $\mathrm{q}-\mathrm{SPC} / \mathrm{FW}^{[49]}$ model, have been introduced.

\subsection{Integrating Polarization into Water Models}

The increase in computational power saw a transition towards increasingly complicated water models with an emphasis on the non-additivity of water-water interac- tions, in particular induction/polarization effects. Polarization is often incorporated explicitly via central or distributed point dipole-dipole polarizabilities, derived from the use of perturbation theory to treat intermolecular forces. ${ }^{[50]}$ Despite the rigorous theoretical background, such an implementation may lead to deceptive results as we shall see in Section 3.1. Furthermore, higher-order multipoles, typically up to quadrupoles, are employed to represent electrostatics instead of point charges in recognition of the anisotropic nature of the electron distribution. This led to more elaborate analytic potentials that required more parameters that would come from a mix of $a b$ initio and experimental spectroscopic data. This class of water models flourished in the 1990s and 2000s when accurate $a b$ initio second-order MøllerPlesset perturbation theory (MP2) and later CCSD(T) calculations become amendable. As the majority of the parameters are monomer properties such as the dipole moment and polarizability, highly accurate $a b$ initio calculations can be performed on the small water monomer system. In some cases, the Vibration-Rotation-Tunnelling (VRT) spectroscopic data was used in the parameterization as they represent information at the atomistic level as opposed to bulk water properties. Using these water models, there would be more studies devoted towards water clusters, underscoring the importance of microscopic understanding of water. As the functional form of these water models grew more complex, it would naturally encompass a larger variety of models and some of the notable water models include the ASP, SAPT and TTM family of water models.

\subsubsection{ASP Family}

The Anisotropic Site Potential with Wormer's dispersion (ASP-W) model, based on Hayes-Stone intermolecular perturbation theory (IMPT), ${ }^{51,52]}$ is one of the earliest rigid water models to adopt higher-order multipoles. ${ }^{[53]}$ For electrostatics, distributed multipoles are present on both the oxygen and hydrogen atomic sites, up to quadrupole and dipole respectively whereas induction is computed at first order (instead of full iteration) using point polarizabilites on oxygen up to quadrupole. Site anisotropy was likewise incorporated into the dispersion and repulsion terms. Further refinements by the same group led to the inclusion of a new charge transfer term, creating the ASP-W2 and ASP-W4 models, used to study the stationary structures of the water dimer. ${ }^{[54]}$ The difference between both models lies in the order of multipoles used with the original multipoles being retained in ASP-W2 and hexadecapole present on each atom in ASP-W4.
The ASP-W functional form was also fitted to the (D2O), VRT spectra, giving rise to the VRT(ASP-W) model.[55] VRT(ASP-W) is the first water model to achieve spectroscopic accuracy, able to reproduce most of the tunnelling barriers in the water dimer. While this is not surprising given the use of same experimental data in constructing the model, it is worthwhile to note that the use of the rigid monomer approximation can still lead to accurate predictions at the atomistic level. Later improvements would give rise to the VRT(ASP-W)II and VRT(ASP-W) III model, where induction is computed to full iteration. ${ }^{[56]}$

\subsubsection{SAPT Family}

Both SAPT-ss and SAPT-pp water models, ${ }^{[57]}$ employing rigid water monomers, were developed based on SymmetryAdapted Perturbation Theory (SAPT). ${ }^{[58]}$ SAPT-ss comprises a site-site form, with a similar placement of sites as TIP4P but instead uses the functional form of the MCY model. Point charges and exponential terms are fitted to 1056 SAPT energies. The SAPT-pp is more complicated, describing the intermolecular interactions using expansions of functions in interatomic vectors and Euler angles, again fitted to the same 1056 SAPT energies.

Due to its complexity, SAPT-pp fell into disuse and the site-site form was evolved to the SAPT-5s model.[59] To reflect the anisotropy of electron distribution, two new symmetry distinct sites representing lone pairs and out-of-plane charges were added, giving a total of five symmetry distinct sites (eight sites in total). An elaborate functional form was adopted using a polynomial-exponential terms to represent exchange-repulsion and an inverse power (6-8-10) series to describe induction and dispersion. Consequently, no iteration of the induced dipole is required in calculating the induction term as it is represented by fitted coefficients. The model's exchange-repulsion parameters are also tuned to better reproduce the water dimer's acceptor tunnelling splitting, giving the revised SAPT-5st.[60]

All the SAPT models mentioned above only contain a pair potential. Thus, threebody SAPT $(\mathrm{HF})$ energies were incorporated into SAPT-5s, giving the SAPT-5s+3B model.[61] This new three-body potential is the first to include functional forms to model three-body exchange effects using a combination of exponential and Legendre polynomial terms. Long-range effects are described using a damped induced dipole model. Later, the SAPT-5s functional form is refitted using SAPT(DFT) energies and this new SDFT-5s model ${ }^{[62]}$ gives more accurate results, attributed to the faster basis set convergence with DFT. 


\subsubsection{TTM Family}

TTM-R, ${ }^{[63]}$ the first of Thole-Type Model (TTM) water models, is based on Thole's idea of using smeared out multipoles to mirror the diffuse picture of electron distribution. ${ }^{64]}$ TTM-R utilises TIP4P-style point charges for electrostatic interactions and an inverse power (6-1012) series to represent dispersion and repulsion. Smeared charges and dipole are present on all atomic sites for induction and intramolecular polarization can occur, accounting for charge transfer. As the TTM-R model consistently over-binds small water clusters, the TTM2-R model was proposed by refitting the inverse power (6-10-12) series to minimum energy pathways connecting the global minimum and other stationary points of the water dimer. ${ }^{[65]}$

Monomer flexibility was then incorporated using the Partridge-Schwenke intramolecular Potential Energy Surface (PES) and Dipole Moment Surface (DMS) ${ }^{[66]}$ resulting in the TTM2-F model, the first water model to properly reproduce an increase in the monomer bending angle in water clusters. ${ }^{[67]}$ A revised TTM2.1-F model, ${ }^{[68]}$ intended for simulations, was proposed by modifying the inverse power (6-10-12) series that decreases unphysically below $2.5 \AA$ as such repulsive regions may be sampled during condensed-phase simulations.

Two unrelated updates, the TTM3-F[69] and TTM4-F model[70] were also reported. Aimed at describing the vibrational spectra of water clusters and bulk water, TTM3-F has modified partial charges to reflect the behaviour that water dissociates to $\mathrm{H}^{+} / \mathrm{OH}^{-}$ in liquid as opposed to radical formation in the gas phase. On the other hand, TTM4-F is reparameterized to better reproduce polarizability surface. Notably, the popular AMOEBA water model uses a Thole-type induction model.[71]

\subsection{Extensive Use of ab initio Data in Water Models}

As $a b$ initio methods matured into reliable tools rivalling experimental accuracy, we ushered in an era of water models empowered by $a b$ initio data. This class of water models relies on high-quality large datasets (in the order of $10^{5}$ data points) of $\operatorname{CCSD}(\mathrm{T})$ energies, the gold standard of quantum chemistry. The water models are deeply rooted in the Many-Body Expansion (MBE) where the total energy of a system can be decomposed into onebody (monomer contribution), two-body (pairwise interactions), three-body contributions and so on. Separate PES are constructed for each of these $k$-body terms by fitting large energy datasets which sample important configuration space encountered in water clusters and during condensed phase simulations. The extensive amount of high-quality data required can only be fulfilled by large volumes of accurate $a b$ initio calculations which only became amendable in recent years. The shift towards large datasets and complicated PES construction techniques stems from the realization that short-range effects such as charge transfer and exchange cannot be accurately described by simple analytic forms. Thus, sufficiently flexible functional forms are required to map the accurate $a b$ initio dataset into high-quality PES for on-the-fly evaluation of energies. Water monomer flexibility is a another common feature in these models although a rigid monomer constraint is often imposed in demanding calculations such as condensed phase simulations and VRT spectra prediction. As a result, these models are mainly focussed on studies of water clusters with few examples of condensed phase simulations. As the construction of these water models is laborious, there were only three families of such $a b$ initio water models, namely the HBB, CC-pol and MB-pol family of water models.

\subsubsection{HBB Family}

The HBB water models describe the PES for each of these $k$-body terms using permutationally invariant polynomials involving interatomic distances, incorporating the permutation symmetry of identical atoms, i.e. the hydrogen and oxygen atoms. This alleviates the steep computational cost in evaluating high-dimensional PES and drastically reduces the number of data points required for fitting the PES. The first HBB0 model uses polynomials of Morse-type exponential functions, fitted to $19805 \mathrm{CCSD}(\mathrm{T}) / \mathrm{AVTZ}$ energies. ${ }^{[72]}$ Like all HBB models, all $N(N-1) / 2$ interatomic distances were used to preserve the permutational symmetry, more than the actual 3N-6 degrees-of-freedom present in the system. In the next revision HBB1, the same functional form is refitted to an additional $10227 \mathrm{CCSD}(\mathrm{T}) / \mathrm{AVTZ}$ energies to better describe the low-energy configuration space below $10000 \mathrm{~cm}^{-1}$.[73] This refitting led the RMS fitting error to drop by a factor of two, suggesting that the quality of the functional form was previously not maximized in HBB0.

A hybrid pair potential was developed in the new HBB2 model, comprising longrange and short-range components. ${ }^{74]}$ The short-range component remains to be described by permutationally invariant polynomials while the long-range component is described using the TTM3-F model. This led to slight improvements in accuracy and large computational savings as the TTM3-F potential is much faster to compute. The $\mathrm{HBB} n$ models only contain a pair potential and cannot be used to describe water clusters where higher-body effects have to be considered. Thus, the WHBB model is introduced where a threebody potential is again constructed using permutationally invariant polynomials, fitted to $40000 \mathrm{MP} 2 / \mathrm{AVTZ}$ energies. ${ }^{[75]}$ Interestingly, it was mentioned that the three-body potential is shorter range than the two-body counterpart and a cutoff was implemented when the maximum $\mathrm{O}-\mathrm{O}$ distance is greater than $8 \AA$. Fourand-higher-body effects are described by induction using the TTM3-F model. For all the water models in the HBB family, the one-body potential is provided by the Partridge-Schwenke intramolecular PES.

\subsubsection{CC-pol Family}

The CC-pol family of water models is the successor of the SAPT family, utilising $a b$ initio energies computed at $\operatorname{CCSD}(\mathrm{T})$ instead of SAPT energies. The first CC-pol model[76] is similar to the SAPT-5s model except that induction is now explicitly iterated instead of using a fitted inverse power series. CC-pol is able to reproduce the water dimer VRT spectra except for the interchange splitting transition, attributed to the rigid monomer approximation.

The CC-pol-8s model revamped the placement of the interaction sites, having eight symmetry distinct sites ( 25 sites in total). ${ }^{[77]}$ The three-dimensional Cartesian space was scanned in regular intervals, followed by finer subgrids to ensure that the most optimal positions were chosen. As only point charges were used (as opposed to higher-order multipoles), the presence of more interaction sites better represents the anisotropy of the electron distribution and led to a four-fold decrease in the fitting errors. A flexible variant, CC-pol-8sf,[78] was developed where monomer contribution to the interaction energy is obtained from an earlier flexible SAPT-5s'fIR water model. ${ }^{[79]}$

Feeling that the order of $10^{5}$ data points is inadequate to build an accurate full 21-dimensional flexible-monomer three-body PES, the authors reverted to a rigid monomer model, consisting of the pair potential CCpol2 and three-body potential CCpol3. ${ }^{[80]} \mathrm{CCpol} 2$ is essentially the same as CC-pol-8s, except that shortrange damping is included to improve the description at very small intermolecular distances as these regions may be sampled during condensed phase simulations. The CCpol3 model, fitted to $71456 \operatorname{CCSD}(\mathrm{T})$ energies, gives improved polarization from the use of three atomic polarization centres, instead of one. Four-and-higher-body interactions are described using a simple polarization model. Surprisingly, the polarization model gives accurate four-body energies to within a few percent, whereas such models are known to have significant errors for three-body interactions. 


\subsubsection{MB-pol Family}

The MB-pol family incorporates many features from the HBB family of $a b$ initio based water models. The prototype HBB2-pol model[81] borrows from the HBB2 model using a hybrid pair potential and the Partridge-Schwenke intramolecular PES. The same HBB2 PES was used for the short-range component of the pair potential while the long-range component was replaced with the TTM4-F model. Furthermore, a three-body hybrid potential is included where the short-range component again incorporates the permutational symmetry, fitted to 8019 CCSD(T) trimer energies, while the long-range counterpart, as well as four-and-higher-body effects, are described by induction in the TTM4-F model. The TTM4-F component greatly reduced the order of the permutationally invariant polynomials and the associated computational cost, making HBB2-pol amendable to condensed phase simulations. TTM4-F was chosen after careful comparison with two other polarizable flexible water models, namely TTM3-F and AMOEBA.

The eventual MB-pol model is described in two papers, detailing the hybrid pair potential[ ${ }^{[2]}$ and higher-body effects separately. ${ }^{[83]}$ The hybrid pair potential MB-pol(2B) was improved with the addition of two new sites to represent the lone pairs of water, which greatly improved the flexibility of the functional form in the short-range component. Thus, the permutationally invariant polynomials now involves intersite distances between the atomic sites and/or the lone pair sites, fitted to $42508 \operatorname{CCSD}(\mathrm{T})$ dimer energies. The three-body potential MB-pol(3B) is described in a similar fashion as in HBB2pol but fitted to a larger dataset of 12347 energies. All long range effects are handled by induction using the TTM4-F model. It was noted that short-range corrections are not required at the four-and-higher-body level, in agreement with CCpol3 authors' observation that a simple polarization model is sufficient.

On a final note, both HBB2-pol and MB-pol are the first water models constructed from extensive CCSD(T) energies dataset to be employed in classical and quantum simulations of liquid water. ${ }^{[84,85]}$ In both instances, many structural and dynamic properties of liquid water under ambient conditions were reproduced, such as the radial distribution functions, bulk water density and diffusion coefficient.

\section{Qualities of a Good Water Model}

After reviewing the plethora of water models shaped by different philosophies, we identified several key features for the proper description of water. They are namely (i) the inclusion of polarizability to account for non-additive effects, (ii) fitting or interpolating energies to account for short-range effects, (iii) incorporation of monomer flexibility, (iv) accounting for quantum effects in simulations and (v) transferability and dissociable water model.

\subsection{Inclusion of Polarizability}

As we witness from the integration of polarization into water models, (Section 2.2) the inclusion of polarizability is crucial in describing the significant manybody inductive effects that arise from the high dipole and polarizability of water. Neglecting polarization effects in empirical point charge water models (such as TIN $n \mathrm{P}$ and SPC models in Section 2.1) prevents an accurate description of virial coefficients, vapour pressures, critical pressure and dielectric constant. ${ }^{[86]}$ The first three quantities involve gas phase properties which are very sensitive to changes in the environment. Clearly, the degree of polarization in the gas phase would differ greatly from that in the condensed phase for which the empirical models are calibrated. Likewise, polarization is required to reproduce the enhanced dipole moment in condensed phase to properly reproduce the dielectric constant.

There are several excellent reviews ${ }^{[30,87-89]}$ on the implementation of polarization as it found importance not only in water models but also in ion solvation, other small molecules and protein simulations. Three methods for incorporating polarization exist, namely fluctuating charge, Drude oscillator and induced point dipole models. While the first two methods have been implemented in water models, (e.g. TIP4P-FQ, SPC-FQ ${ }^{[40]}$ for fluctuating charge and SWM4-DP[90] for Drude oscillator) the induced point dipole model remains the most implemented for water models. In fact, the ASP, SAPT and TTM families of water models in Section 2.2 all use some kind of induced point dipole model. In principle, higher-order multipoles such as the quadrupole can also be induced as seen in the ASP water models but they see little action elsewhere (SAPT and TTM families only involve inducible dipole) perhaps due to the laborious theoretical expressions involved. While the introduction of inducible dipole models is increasingly prevalent, Guillot cautions that poor implementation can lead to deceptive results. ${ }^{[29]}$ The induced dipole moment is given as the product of the polarizability with the electric field. The electric field is often represented by the point charges/multipoles present in the model and this may be inadequate if higher-order multipoles are not considered.[91]
Furthermore, there is also dipole-quadrupole and quadrupole-quadrupole polarizabilities which are often neglected and these inductive effects can be significant given that water has a strong quadrupole moment.

Finally, Thole ${ }^{[40]}$ and Applequist et $a l .{ }^{[92]}$ have pointed out that the point induced dipole moment may become infinite at small distances, which is commonly known as the 'polarization catastrophe'. This can be avoided by screening the dipole-dipole interaction at short distances, either using a Tang-Toennies damping function ${ }^{[93]}$ as seen in the ASP and SAPT models or using smeared out charges and dipoles in TTM models. This screening is an indication that point multipoles cannot properly describe the electronic distribution at small distances, underscoring the importance of accounting for short-range effects.

\subsection{Short-range Effects}

At short intermolecular distances $R$, the power series expansion of inverse $R$ which defines the point multipole diverges, causing the failure of point multipoles at short-range. Furthermore, there is a charge penetration effect as the electrons are 'not fully felt' within the electron cloud. Physically, this can be interpreted as the unrealistic representation of the electronic distribution as if it was concentrated at a point. Possible remedies include the use of damping functions or smeared out multipoles as seen in Section 2.2 as well as partitioning the electronic distribution using distributed multipoles. ${ }^{[50]}$ Despite these corrections, other short-range interactions such as exchange-repulsion and charge transfer have to be explicitly accounted for. The distinction between short-range and long-range interactions (electrostatic, induction and dispersion) is rooted in their different physical character where shortrange effects vary exponentially with intermolecular distance while long-range effects behave as some inverse power of intermolecular distance. [50] Thus, it would be prudent to separate the total interaction energy into short-range and long-range components due to their intrinsically different nature as seen in the HBB2, WHBB, HBB2-pol and MB-pol water models.

Unfortunately, unlike long-range interactions which have well-defined formulae based on IMPT, no exact analytic form exists for short-range interactions. Otherwise, high quality ab initio methods which can describe these subtle shortrange effects up to any desired numerical precision would have been developed in vain. For the ASP, SAPT and TTM families of models, short-range exchange-repulsion effects were modelled by simple exponential and/or polynomial-exponential terms. 
As these approaches proved inadequate, large $a b$ initio data sets are fitted to more complicated functional forms to accurately describe these exchange-repulsion effects (Section 2.3). Currently, two such functional forms have been implemented. The permutationally invariant polynomials in $\mathrm{HBB}$ and MB-pol families of models incorporate the permutational symmetry of identical nuclei into exponential terms involving interatomic distances. On the other hand, CC-pol models uses simple polynomial-exponential terms but applied between a large number of symmetry-distinct sites, greatly increasing the flexibility of the functional form. Inevitably, both methods incorporate some form of symmetry which serves to alleviate the high computational cost. Furthermore, both methods involve fitting of the coefficients of the terms from $a b$ initio data. An alternative to fitting methods would be interpolation methods. Examples include Shepard interpolation ${ }^{[94,95]}$ and Interpolating Moving Least Square ${ }^{[96,97]}$ as well as simpler interpolating methods such as cubic splines. While interpolation methods ensure that the PES passes exactly through the dataset, care has to be taken that the asymptotic behaviour of the PES is enforced in interpolating models which are otherwise naturally incorporated into the functional forms used in fitting models. Nonetheless, it would be interesting to see new ab initio based water models based on interpolation methods and compare their accuracy with existing models.

Another essential formalism employed to describe short-range effects would be the Many-Body Expansion (MBE). Without the use of MBE, the dimensionality of the system would be too large for any fitting or interpolation method to be feasible. Instead, using the MBE, large water clusters or even bulk water can be decomposed into many-body contributions, truncated at the four-body level. However, basis set superposition effects causes poor convergence of the MBE when diffuse basis functions are involved[98] and these diffuse functions are crucial in accurately describing the hydrogen bonding between water molecules.

\subsection{Monomer Flexibility}

In the MBE formalism, the one-body contribution would correspond to intramolecular distortions of water monomer. Due to computational limitations, pioneering empirical water models often employ rigid monomers. While later models would comprise of flexible monomers, a rigid monomer approximation is still preferred for computationally demanding calculations such as spectra prediction and condensed phase simulations. Also, a large dataset is required to fit flexible monomer potentials which can disfavour their use as seen in the CCpol2 and CCpol3 water models. It is recommended that the vibrational averaged geometry be used over the equilibrium geometry when a rigid monomer approximation is necessary.

Monomer flexibility is integral in the atomistic understanding of water as subtle changes in bond lengths and angles can affect the predicted energetics and VRT spectra of water clusters. The first water models to include flexible monomers use quadratic terms to describe the stretching and bending motions, modelling the vibrational modes as harmonic oscillators. This is overly simplistic in dealing with the quantum mechanical effects that arises when the electron clouds of the two hydrogens overlap during the bending motion. Thus, more sophisticated intramolecular PES were constructed, the most popular being the Partridge-Schwenke intramolecular PES, which is used in the TTM, HBB and MB-pol families of water models. The Partridge-Schwenke PES is also accompanied with an intramolecular DMS which supplies the dipole moment required in the calculation of long-range interactions. This could be the reason why higher-order multipoles are not involved in the longrange components of these models as an accurate quadrupole moment surface do not exist yet.

It is important to realize that these intramolecular vibrations are quantum mechanical in nature and their treatment within classical simulations may not yield satisfactory results. ${ }^{[99-101]}$ The representative example would be the harmonic oscillator where the classical probability would be greatest away from the equilibrium while the quantum counterpart has the maximum probability at the equilibrium position. Thus, flexible water models should be simulated using methods that incorporate quantum effects.

\subsection{Nuclear Quantum Effects}

Nuclear quantum effects and monomer flexibility are intertwined since the motions of the nuclei obey the laws of quantum mechanics rather than the classical counterpart. This is especially so for water due to the presence of the light hydrogen nuclei and extensive hydrogen bonding, both of which exhibit strong nuclear quantum effects. Thus, processes involving the hydrogen nuclei such as Grotthuss proton shuttling ${ }^{[102]}$ require nuclear quantum effects to be accounted for. ${ }^{[103]}$

Furthermore, disregarding nuclear quantum effects can lead to poor description of the heat capacity of both liquid and solid water ${ }^{[104,105]}$ and low-temperature properties such as the densities of ice polymorphs. ${ }^{[99]}$ In addition, when nuclear quantum effects are neglected, isotopic effects cannot be probed, which can have a significant influence in bulk properties. For example, the enthalpy of vaporization is a measure of the strength of the hydrogen bonding within liquid water. Classically, there should be no isotopic effects present. However, it has been shown experimentally that the isotopic effects on the vaporization enthalpy is important, increasing by $0.4 \mathrm{kcal} \mathrm{mol}^{-1}$ from water to tritiated water. ${ }^{[86]}$

A variety of quantum simulation methods exist and some of the computational methodologies have been reviewed.[106] The most commonly employed method would be Path Integral Molecular Dynamics (PIMD), ${ }^{107-109]}$ which exploits the isomorphism between the quantum partition function expressed in path integral formalism and the classical partition function of a ring-polymer. This isomorphism provides a way to sample the quantum nuclear configuration through modifications of the classical MD technique. Other quantum simulation methods would include Path Integral Monte Carlo (PIMC), [110,111] Path Integral Hybrid Monte Carlo (PIHMC), [107,112,113] Centroid Molecular Dynamics (CMD) [114-118] and Ring Polymer Molecular Dynamics (RPMD). [119,120]

While PIMD simulations have been performed for the HBB2-pol and MB-pol $a b$ initio based models at ambient conditions, ${ }^{[84,85]}$ extreme conditions (low temperatures, critical point) have not been explored to elucidate the anomalous behaviour of water. On a side note, studies on the quantum effects of water performed on empirical water models such as TIP4P should be interpreted with caution. As such water models are parameterized to reproduce experimental values using classical simulations, quantum effects are included in these models in an effective manner. Thus, performing quantum simulations on these water models to investigate quantum effects seems counterproductive unless the model has been reparameterized for such purposes.

\subsection{Transferability and Ability to Dissociate}

While less discussed in literature, it is ideal to develop a water model to be used outside pure water systems for applications such as explicit solvation of proteins. The empirical and polarizable models (Section 2.1 and 2.2) are highly transferable due to the use of point multipoles which share the same functional form regardless of the molecular species. This is not the case for $a b$ initio based water models (Section 2.3) that rely on the MBE as new PES have to be constructed for new combinations of $k$-body interactions.

Finally, very few models in literature 
are able to dissociate into $\mathrm{H}^{+} / \mathrm{OH}^{-}$ions. Water dissociation is difficult to handle as the products (charged ions) are very different from the reactant (neutral molecules). This is complicated by the fact that water dissociates homolytically into radicals in the gas phase. It would be optimal to use on-the-fly $a b$ initio simulation techniques such as Car-Parrinello Molecular Dynamics (CPMD) ${ }^{[121]}$ to study water dissociation as these $a b$ initio methods do not make any distinction between $\mathrm{H}^{+} / \mathrm{OH}^{-}$ions and neutral water molecules.

\section{Concluding Remarks and Outlook}

The scene of water modelling remains a vibrant one, especially in the last 15 years where countless water models of distinct modelling philosophies have been developed with the sole aim to better understand this mysterious liquid. The strengths and (more often) inadequacies of these water models have provided useful information on the essential ingredients for the making of a universal water model.

It is only very recently, with the extensive use of $a b$ initio data and availability of quantum simulations, that water models possess the right qualities to accurately describe water at both the microscopic and macroscopic level. Yet, there still leaves room for development, in seeking new ways to describe short-range effects using interpolation techniques and employing higher-order multipoles in long-range interactions so that more of the configuration space can be described cheaply.

Nonetheless, it is due time to put these state-of-the-art water models to more rigorous tests to reproduce experimental results at extreme conditions. If these water models were to succeed at these trials, then perhaps it would be possible to explain the many anomalies of water, fulfilling the role of computations in assisting experiments to dispel confusion and eventually pushing the boundaries of science.

\section{Acknowledgements}

The authors thank the National University of Singapore's support from the Academic Research Fund, grant number R-143-000-549112.

Received: January 2, 2015

[1] F. Franks, 'Water: A Matrix of Life', $2^{\text {nd }}$ ed., Royal Society Of Chemistry, Cambridge, England, 2000.

[2] M. J. Tait, F. Franks, Nature 1971, 230, 91.

[3] M. Chaplin, Nature Rev. 2006, 7, 861.

[4] P. Ball, Chem. Rev. 2008, 108, 74.

[5] M. S. Cheung, A. E. Garcia, J. N. Onuchic, Proc. Natl. Acad. Sci. USA 2002, 99, 685.

[6] M. Fuxreiter, M. Mezei, I. Simon, R. Osman, Biophys. J. 2005, 89, 903.
[7] G. Tinetti, A. Vidal-Madjar, M.-C. Liang, J.P. Beaulieu, Y. Yung, S. Carey, R. J. Barber, J. Tennyson, I. Ribas, N. Allard, G. E. Ballester, D. K. Sing, F. Selsis, Nature 2007, 448, 169.

[8] F. Robert, Science 2001, 293, 1056.

[9] N. Sakamoto, Y. Seto, S. Itoh, K. Kuramoto, K. Fujino, K. Nagashima, A. N. Krot, H. Yurimoto, Science 2007, 317, 231

[10] P. Jenniskens, D. F. Blake, Science 1994, 265, 753.

[11] F. Franks, in 'Water: A Comprehensive Treatise Vol 1 Physics and Physical Chemistry of Water', Ed. F. Franks, Plenum Press, New York, USA, 1972.

[12] P. H. Poole, U. E. F. Sciortino, H. E. Stanley, Nature 1992, 360, 324

[13] H. E. Stanley, S. V. Buldyrev, M. Canpolat, S. Havlin, O. Mishima, M. R. Sadr-Lahijany, A. Scala, F. Starr, Physica D 1999, 133, 453.

[14] F. X. Prielmeier, E. W. Lang, R. J. Speedy, H.D. Lüdemann, Phys. Rev. Lett. 1987, 59, 1128.

[15] R. S. Smith, B. D. Kay, Nature 1999, 398, 788.

[16] L. Liu, S.-H. Chen, A. Faraone, C.-W. Yen, C.-Y. Mou, Phys. Rev. Lett. 2005, 95, 117802.

[17] C. G. Salzmann, P. G. Radaelli, A. Hallbrucker, E. Mayer, J. L. Finney, Science 2006, 311, 1758.

[18] C. G. Salzmann, P. G. Radaelli, E. Mayer, J. L. Finney, Phys. Rev. Lett. 2009, 103, 105701.

[19] I. Ohmine, H. Tanaka, Chem. Rev. 1993, 93, 2545.

[20] W. M. Latimer, W. H. Rodebush, J. Am. Chem. Soc. 1920, 42, 1419.

[21] B. J. Smith, D. J. Swanton, J. A. Pople, III, H. F. S.; L. Radom, J. Chem. Phys. 1990, 92, 1240.

[22] G. S. Tschumper, M. L. Leininger, B. C. Hoffman, E. F. Valeev, III, H. F. S.; M. Quack, J. Chem. Phys. 2002, 116, 690.

[23] J. R. Lane, J. Chem. Theory Comput. 2013, 9, 316.

[24] E. R. Batista, S. S. Xantheas, J. Chem. Phys. 1998, 109, 4546 .

[25] B. Guillot, J. Mol. Liq. 2002, 101, 219.

[26] J. D. Bernal, R. H. Fowler, J. Chem. Phys. 1933, 1,515 .

[27] J. A. Barker, R. O. Watts, Chem. Phys. Lett. 1969, 3, 144.

[28] A. Rahman, F. H. Stillinger, J. Chem. Phys. 1971, 55,3336

[29] B. Guillot, J. Mol. Liq. 2002, 101, 219.

[30] T. A. Halgren, W. Damm, Curr. Opin. Struct. Biol. 2001, 11, 236.

[31] J. L. Finney, J. Mol. Liq. 2001, 90, 303.

[32] K. Szalewicz, C. Leforestier, A. van der Avoird, Chem. Phys. Lett. 2009, 482, 1.

[33] O. Demerdash, E.-H. Yap, T. Head-Gordon, Annu. Rev. Phys. Chem. 2014, 65, 149.

[34] K. Raghavachari, G. W. Trucks, J. A. Pople, M. Head-Gordon, Chem. Phys. Lett. 1989, 157, 479.

[35] T. H. Dunning, Jr., J. Chem. Phys. 1989, 90, 1007.

[36] A. D. MacKerell, Jr., D. Bashford, M. Bellott, R. L. Dunbrack, Jr., J. D. Evanseck, M. J. Field, S. Fischer, J. Gao, H. Guo, S. Ha, D. JosephMcCarthy, L. Kuchnir, K. Kuczera, F. T. K. Lau, C. Mattos, S. Michnick, T. Ngo, D. T. Nguyen, B. Prodhom, W. E. Reiher, III, B. Roux, M. Schlenkrich, J. C. Smith, R. Stote, J. Straub, M. Watanabe, J. Wiurkiewicz-Kuczera, D. Yin, M. Karplus, J. Phys. Chem. B 1998, 102, 3586.

[37] H. Popkie, H. Kistenmacher, E. Clementi, J. Chem. Phys. 1973, 59, 1325.

[38] W. L. Jorgensen, J. Am. Chem. Soc. 1981, 103, 335.

[39] W. L. Jorgensen, J. Chandrasekhar, J. D. Madura, R. W. Impey, M. L. Klein, J. Chem. Phys. 1983, 79, 926.

[40] S. W. Rick, S. J. Stuart, B. J. Berne, J. Chem. Phys. 1994, 101, 6141.

[41] M. W. Mahoney, W. L. Jorgensen, J. Chem. Phys. 2000, 112, 8910.
[42] M. W. Mahoney, W. L. Jorgensen, J. Chem. Phys. 2001, 115, 10758 .

[43] H. W. Horn, W. C. Swope, J. W. Pitera, J. D. Madura, T. J. Dick, G. L. Hura, J. Chem. Phys. 2004, 120, 9665.

[44] J. L. F. Abascal, C. Vega, J. Chem. Phys. 2005, $123,234505$.

[45] S. Habershon, T. E. Markland, D. E. Manolopoulos, J. Chem. Phys. 2009, 131, 024501 .

[46] H. J. C. Berendsen, J. P. M. Postma, W. F. von Gunstaren, J. Hermans, in 'Intermolecular Forces', Ed. B. Pullman, Reidel, Dordrecht, Holland, 1981, pp 331-342.

[47] H. J. C. Berendsen, J. R. Grigera, T. P. Straatsma, J. Phys. Chem. 1987, 91, 6269.

[48] Y. Wu, H. L. Tepper, G. A. Voth, J. Chem. Phys. 2006, 124, 024503.

[49] F. Paesani, W. Zhang, D. A. Case, T. E. Cheatham III, G. A. Voth, J. Chem. Phys. 2006, 125, 184507.

[50] A. J. Stone, 'The Theory of Intermolecular Forces', 2nd ed., Oxford University Press, Oxford, England, 2013.

[51] I. C. Hayes, A. J. Stone, Mol. Phys. 1984, 53 , 69.

[52] I. C. Hayes, A. J. Stone, Mol. Phys. 1984, 53, 83.

[53] C. Millot, A. J. Stone, Mol. Phys. 1992, 77, 439.

[54] C. Millot, J.-C. Soetens, M. T. C. M. Costa, M. P. Hodges, A. J. Stone, J. Phys. Chem. A 1998, 102, 754 .

[55] R. S. Fellers, C. Leforestier, L. B. Braly, M. G Brown, R. J. Saykally, Science 1999, 284, 945.

[56] N. Goldman, R. S. Fellers, M. G. Brown, L. B. Braly, C. J. Keoshian, C. Leforestier, R. J. Saykally, J. Chem. Phys. 2002, 116, 10148.

[57] E. M. Mas, K. Szalewicz, R. Bukowski, B. Jeziorski, J. Chem. Phys. 1997, 107, 4207.

[58] B. Jeziorski, R. Moszynski, K. Szalewicz, Chem. Rev. 1994, 94, 1887.

[59] E. M. Mas, R. Bukowski, K. Szalewicz, G. C. Groenenboom, P. E. S. Wormer, A. van der Avoird, J. Chem. Phys. 2000, 113, 6687.

[60] G. C. Groenenboom, P. E. S. Wormer, A. van der Avoird, E. M. Mas, R. Bukowski, K. Szalewicz, J. Chem. Phys. 2000, 113, 6702.

[61] E. M. Mas, R. Bukowski, K. Szalewicz, J. Chem. Phys. 2003, 118, 4386.

[62] R. Bukowski, K. Szalewicz, G. Groenenboom, A. van der Avoird, J. Chem. Phys. 2006, 125, 044301.

[63] C. J. Burnham, J. Li, S. S. Xantheas, M. Leslie, J. Chem. Phys. 1999, 110, 4566.

[64] B. T. Thole, Chem. Phys. 1981, 59, 341.

[65] C. J. Burnham, S. S. Xantheas, J. Chem. Phys. 2002, 116, 1500 .

[66] H. Partridge, D. W. Schwenke, J. Chem. Phys. 1997, 106, 4618.

[67] C. J. Burnham, S. S. Xantheas, J. Chem. Phys. 2002, 116, 5115

[68] G. S. Fanourgakis, S. S. Xantheas, J. Phys. Chem. A 2006, 110, 4100.

[69] G. S. Fanourgakis, S. S. Xantheas, J. Chem. Phys. 2008, 128, 074506

[70] C. J. Burnham, D. J. Anick, P. K. Mankoo, G. F. Reiter, J. Chem. Phys. 2008, 128, 154519.

[71] P. Ren, J. W. Ponder, J. Phys. Chem. B 2003, 107, 5933.

[72] X. Huang, B. J. Braams, J. M. Bowman, J. Phys. Chem. A 2006, 110, 445.

[73] X. Huang, B. J. Braams, J. M. Bowman, R. E A. Kelly, J. Tennyson, G. C. Groenenboom, A. van der Avoird, J. Chem. Phys. 2008, 128, 034312.

[74] A. Shank, Y. Wang, A. Kaledin, B. J. Braams, J. M. Bowman, J. Chem. Phys. 2009, 130, 144314.

[75] Y. Wang, X. Huang, B. C. Shepler, B. J. Braams, J. M. Bowman, J. Chem. Phys. 2011, 134, 094509.

[76] R. Bukowski, K. Szalewicz, G. C. Groenenboom, A. van der Avoird, J. Chem. Phys. 2008, 128, 094313. 
[77] W. Cencek, K. Szalewicz, C. Leforestier, R. van Harrevelt, A. van der Avoird, Phys. Chem. Chem. Phys. 2008, 10, 4716.

[78] C. Leforestier, K. Szalewicz, A. van der Avoird, J. Chem. Phys. 2012, 137, 014305.

[79] K. Szalewicz, G. Murdachaew, R. Bukowski, O. Akin-Ojo, C. Leforestier, in 'Lecture Series on Computer and Computational Science: ICCMSE 2006', Eds. G. Maroulis, T. Simos, Brill Academic, Leiden, 2006, pp 482-491.

[80] U. Góra, W. Cencek, R. Podeszwa, A. van der Avoird, K. Szalewicz, J. Chem. Phys. 2014, 140, 194101.

[81] G. R. Medders, V. Babin, F. A. Paesani, J. Chem. Theory Comput. 2013, 9, 1103.

[82] V. Babin, C. Leforestier, F. Paesani, J. Chem. Theory Comput. 2013, 9, 5395.

[83] V. Babin, G. R. Medders, F. Paesani, J. Chem. Theory Comput. 2014, 10, 1599.

[84] V. Babin, G. R. Medders, F. Paesani, J. Phys. Chem. Lett. 2012, 3, 3765.

[85] G. R. Medders, V. Babin, F. Paesani, J. Chem Theory Comput. 2014, 10, 2906.

[86] C. Vega, J. L. F. Abascal, Phys. Chem. Chem. Phys. 2011, 13, 19663

[87] S. W. Rick, S. J. Stuart, Rev. Comp. Chem. 2002, 18, 89.

[88] J. W. Ponder, D. A. Case, Adv. Prot. Chem. 2003, 66, 27.

[89] P. E. M. Lopes, B. Roux, A. D. MacKerell Jr., Theor. Chem. Acc. 2009, 124, 11
[90] G. Lamoureux, A. D. MacKerell Jr., B. Roux, J. Chem. Phys. 2003, 119, 5185.

[91] S. Cardamone, T. J. Hughes, P. L. A. Popelier, Phys. Chem. Chem. Phys. 2014, 16, 10367.

[92] J. Applequist, J. R. Carl, K.-K. Fung, J. Am. Chem. Soc. 1972, 94, 2952.

[93] K. T. Tang, J. P. Toennies, J. Chem. Phys. 1984, $80,3726$.

[94] J. Ischtwan, M. A. Collins, J. Chem. Phys. 1994, 100, 8080 .

[95] R. P. A. Bettens, M. A. Collins, J. Chem. Phys. 1999, $111,816$.

[96] G. G. Maisuradze, D. L. Thompson, A. F Wagner, M. Minkoff, J. Chem. Phys. 2003, 119, 10002.

[97] Y. Guo, A. Kawano, D. L. Thompson, A. F. Wagner, M. Minkoff, J. Chem. Phys. 2004, 121, 5091.

[98] J. F. Ouyang, M. W. Cvitkovic, R. P. A. Bettens, J. Chem. Theory Comput. 2014, 10, 3699.

[99] C. McBride, C. Vega, E. G. Noya, R. Ramírez, L. M. Sesé, J. Chem. Phys. 2009, 131, 024506.

[100] E. G. Noya, C. Vega, L. M. Sesé, R. Ramírez, J. Chem. Phys. 2009, 131, 124518.

[101] M. M. Conde, C. Vega, C. McBride, E. G. Noya, R. Ramírez, L. M. Sesé, J. Chem. Phys. 2010, 132, 114503 .

[102] N. Agmon, Chem. Phys. Lett. 1995, 244, 456.

[103] C. Knight, G. A. Voth, Acc. Chem. Res. 2012, 45, 101.

[104] W. Shinoda, M. Shiga, Phys. Rev. E 2005, 71, 041204.
[105] M. Shiga, W. Shinoda, J. Chem. Phys. 2005, 123, 134502.

[106] F. Paesani, G. A. Voth, J. Phys. Chem. B 2009, $113,5702$.

[107] M. E. Tuckerman, B. J. Berne, G. J. Martyna, M. L. Klein, J. Chem. Phys. 1993, 99, 2796.

[108] D. Marx, M. Parrinello, J. Chem. Phys. 1995, 104, 4077.

[109] M. Shiga, M. Tachikawa, S. Miura, J. Chem. Phys. 2001, 115, 9149 .

[110] J. A. Barker, J. Chem. Phys. 1979, 70, 2914.

[111] R. A. Kuharski, P. J. Rossky, J. Chem. Phys. 1985, 82,5164

[112] S. Miura, J. Chem. Phys. 2007, 126, 114308.

[113] K. Suzuki, M. Tachikawa, M. Shiga, J. Chem. Phys. 2010, 132, 144108.

[114] J. Cao, G. A. Voth, J. Chem. Phys. 1994, 100, 5093.

[115] J. Cao, G. A. Voth, J. Chem. Phys. 1994, 100, 5106.

[116] J. Cao, G. A. Voth, J. Chem. Phys. 1994, 101, 6157.

[117] J. Cao, G. A. Voth, J. Chem. Phys. 1994, 101, 6168.

[118] J. Cao, G. A. Voth, J. Chem. Phys. 1994, 101, 6184.

[119] I. R. Criag, D. E. Manolopoulos, J. Chem. Phys. 2004, 121, 3368

[120] B. J. Braams, D. E. Manolopoulos, J. Chem. Phys. 2006, 125, 124105.

[121] K. Laasonen, M. Sprik, M. Parrinello, R. Car, J. Chem. Phys. 1993, 99, 9080. 